\title{
Adhesion hysteresis in Dynamic Atomic Force Microscopy
}

\author{
Mariana Köber ${ }^{1}$, Enrique Sahagún ${ }^{2}$, Martina Fuss $^{1}$, Fernando Briones ${ }^{1}$, Mónica Luna ${ }^{*}, 1$, \\ and Juan José Sáenz ${ }^{\text {, }}$ \\ ${ }^{1}$ Instituto de Microelectrónica de Madrid (IMM-CSIC), Isaac Newton 8, 28760 Tres Cantos, Madrid, Spain \\ 2 Departamento de Física de la Materia Condensada, Universidad Autónoma de Madrid, 28049 Madrid, \\ Spain \\ * Corresponding author: e-mail mluna@imm.cnm.csic.es , Phone: +34 91 8372225, Fax: +34 918060701 \\ ** Corresponding author: e-mail juanjo.saenz@uam.es , Phone: +34 91 4973804, Fax: +34 914973961
}

\begin{abstract}
The effects of adhesion hysteresis in the dynamic-dissipation curves measured in amplitude-modulation atomic force microscopy are discussed. Hysteresis in the interaction forces is shown to modify the dynamics of the cantilever leading to different power dissipation curves in the repulsive and attractive regimes. Experimental results together with numerical simulations show that power dissipation, as measured in force microscopy, is not always proportional to the energy dissipated in the tip-sample interaction process.
\end{abstract}

1 Introduction Most real processes that occur when two materials are brought together involve energy dissipation or hysteretic phenomena. The understanding of the microscopic mechanisms of energy dissipation is then fundamentally relevant for a large variety of basic and applied problems (adhesion, contact formation, friction, wear...) and has motivated extensive theoretical and experimental efforts over a century [1]. The development of the atomic force microscope (AFM) opened a new way to study energy losses at nanometer scales. Phase shift variations, measured by recording the phase lag of the cantilever oscillation relative to the driving signal, are directly linked to energy dissipation processes [2-4]. At fixed feedback amplitude, phase contrast images are proportional to power dissipation maps which can potentially be translated into maps of substrate physicochemical properties [5].

Amplitude-modulation AFM (AM-AFM), also known as tapping mode AFM, has been shown to be a powerful tool for qualitative compositional/dissipation mapping. AM-AFM dissipation spectroscopy, based on the analysis of the dissipated power as a function of the cantilever oscillation amplitude, has been proposed as a way to identify specific energy-dissipation processes behind the compositional contrast [5]. However, despite 
of these important advances, the actual link between power dissipation and nanoscale surface properties is not well understood.

As a general approach, power dissipation in AM-AFM is naturally considered synonymous of energy dissipated per cycle. This energy can then be directly related to specific tip-surface interaction processes, number of chemical bonds, etc. In striking contrast to this apparently natural argument, we show that the time-averaged dissipated power is not always proportional to the instantaneous energy dissipated in the tip-sample interaction for each oscillation due to a beating phenomenon where the interaction is occasionally dissipative.

In this Letter we present AM-AFM dynamic-dissipation experiments performed on mica samples (at $0 \%$ relative humidity) together with numerical calculations based on a point mass model. An excellent agreement between experiments and calculations is obtained assuming a simple mechanical hysteresis model where the energy dissipated in every contact process is a fixed quantity. Due to the bistable motion of the cantilever $[6,7]$, the dissipated power strongly depends on the AFM operating regime. While in the repulsive (high amplitude) regime power dissipation is constant (as expected from a fixed energy loss per cycle), in the attractive (low amplitude) regime both experimental and calculated power dissipation strongly depend on the cantilever oscillation amplitude.

2 Experimental setup Measurements were performed at room temperature with an AFM (Nanotec Electrónica Electrónica, Spain) kept in an atmosphere of $2 \pm 0.5 \%$ relative humidity value in order to avoid dissipation due to capillary condensation [8]. The mica substrate was cleaved immediately prior to the experiment. A monocrystalline silicon cantilever (Nanosensors, Nanoworld, Switzerland) with a spring constant of $k=3.2 \mathrm{~N} / \mathrm{m}$, determined following Ref. [9], was driven at its free resonance frequency $\left(v_{0}=85.3 \mathrm{kHz}\right)$. The dissipated power $P_{\text {dis }}$ is related to the oscillation amplitude $(A)$ and the phase shift between the driving excitation and its response $(\varphi)$ through [2]

$$
P_{\text {dis }}=\frac{k A_{0}^{2} \pi v_{0}}{Q}\left(\frac{A}{A_{0}} \sin \varphi-\left(\frac{A}{A_{0}}\right)^{2}\right),
$$

$A_{o}$ being the free oscillation amplitude of the cantilever and $Q$ the quality factor. In order to obtain $P_{\text {dis }}, Q$ must be determined close enough to the surface to include film damping effects [2]. Therefore an oscillation spectrum (Fig. 1) was recorded at a tipsample distance $D_{t s}$ which was just a few nanometres larger than the free oscillation amplitude. A Lorenztian fit to the experimental data resulted in a $Q$ of 160 . Dynamic dissipation curves (see Fig. 2a and 2c) were obtained approaching the oscillating cantilever towards the surface while recording both $A$ and $\varphi$ for different free oscillation amplitudes $A_{O}$ (ranging from 11 to $84 \mathrm{~nm}$ ). 


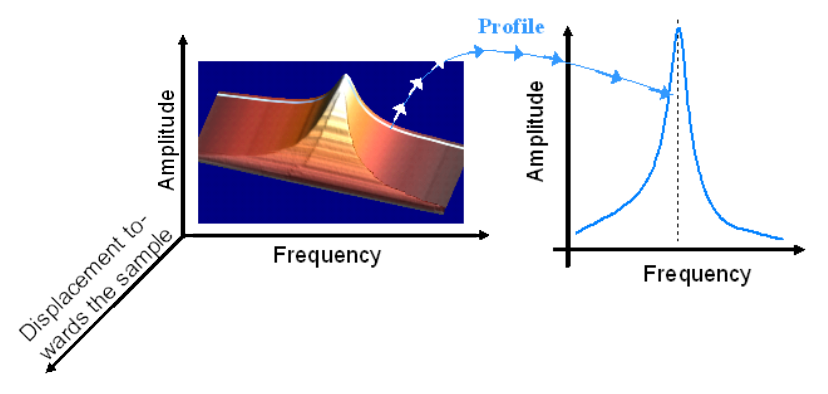

Figure 1 Amplitude vs. frequency curves are recorded while the tip approaches the surface (left). The quality factor $Q$ is obtained from a Lorentzian fit close to the region in which the amplitude starts to diminish due to tip-sample interactions (right).

3 Theoretical model The dynamics of the tip-cantilever ensemble is approximately described as a driven anharmonic oscillator including the cantilever elastic response, the hydrodynamic damping with the medium and the tip-sample interaction forces $F_{t s}$ (including both van der Waals and Dejarguin-Muller-Toporov [10] contact forces as discussed in Ref. [11]). Dissipation is taken into account by adding a hysteretic force $F_{\text {hys }}$. In absence of capillary forces, possible mechanisms that can give rise to hysteresis in an adhesion/decohesion process include plastic or viscoelastic deformations, chemical and mechanical hysteresis [12]. Molecular dynamics (MD) simulations [13] predicted hysteresis in the force versus tip-sample distance related to intrinsic mechanical instabilities at atomic scale. As the tip approaches the sample the interaction is essentially given by the attractive conservative forces (i.e. $F_{\text {hys }}=0$ ). Just before contact, there is a sudden jump of the interaction force due to the formation of an atomic scale connective neck and, as the tip retracts, there is an additional adhesive force which drops approximately linear in a few interatomic distances $\left(D_{0}\right)$. It is worth noticing that this behaviour associated with the formation and rupture of a solid neck [13] is similar (except for some oscillations due to atomic rearrangements) to the one discussed in the context of capillary induced liquid bridges [8]. As a simple approach, we then consider a linear adhesive force (when the tip retracts)

$$
F_{h y s} \approx \frac{2 \Delta E}{D_{0}^{2}}\left(D_{t s}-D_{0}\right) \quad\left(\text { for } \mathrm{D}_{\mathrm{ts}}<\mathrm{D}_{0}\right),
$$

where $\Delta E$ is the energy dissipated in the contact process. The simulations were performed by solving numerically the cantilever's equation of motion at fixed excitation frequency $v_{0}$. The values for $k, v_{0}$ and $Q$ were set to the values determined in the experiments. The tip radius $R$ was set to $15 \mathrm{~nm}$, the Hamaker constant for mica to $10^{-20} \mathrm{~J}$ and the effective Young Modulus to $54 \mathrm{GPa}$. The tip-sample contact takes place when $D_{t s}$ is of the order of an intermolecular distance $a_{0}(0.25 \mathrm{~nm})$. Following typical MD results [13], $D_{0}$ was assumed to be $3 a_{0}$ while $\Delta E$ was a fit parameter to experimental results. 
4 Results and discussion Previous works [14] had focused on the effect of adhesion hysteresis in the frequency modulation mode (FM-AFM), where the resonance frequency shift is recorded while keeping $\varphi=90^{\circ}$. Assuming that the dissipation takes place in each oscillation cycle [14], $P_{\text {dis }}$ would be proportional to the frequency. In AM-AFM we would then have $P_{\text {dis }}=\Delta E v_{0}$ which, for fixed $\Delta E$ and $v_{0}$, would be constant and independent of the amplitude. However, as discussed below, a small beating in the cantilever motion [15] (sketched in the inset of Fig. 2) can lead to an unexpected behaviour of $P_{\text {dis }}$.

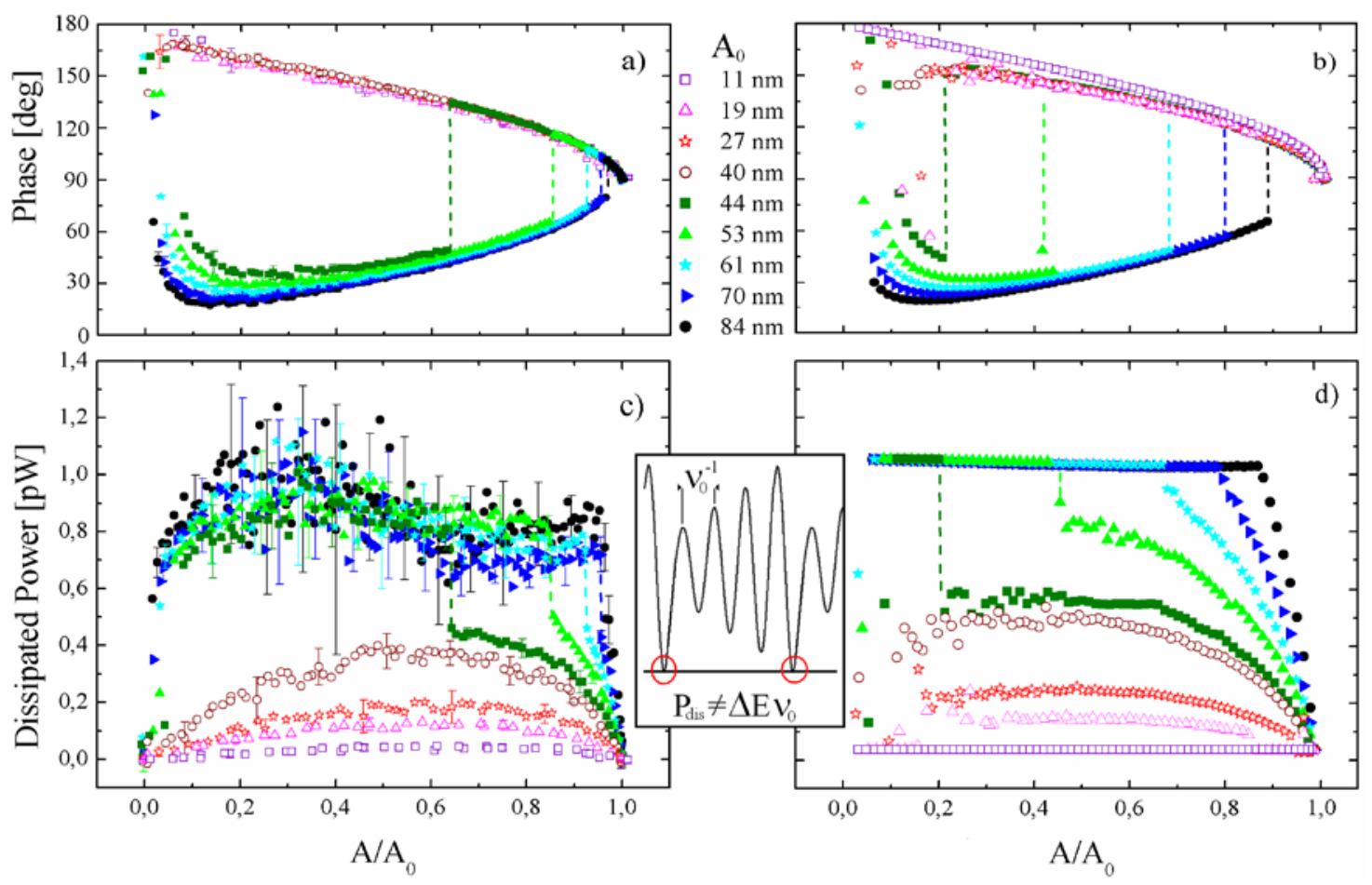

Figure 2a-d Experimental (2a) and calculated (2b) phase shift and experimental (2c) and theoretical (2d) dissipated power versus the oscillation amplitude obtained as the tip-sample distance is reduced. Symbols correspond to different free oscillation amplitudes $A_{0}$. Experimental data were smoothed (average over 10 data points) and the standard deviation is shown. The time evolution of the tip-sample distance in the attractive regime is sketched in the central inset.

Figure 2 shows the phase and the dissipated power, experimentally measured ( $a$ and c) and theoretically calculated ( $b$ and $d$ ), versus the normalized amplitude, as the tip approaches the surface. A remarkable agreement between experiments and calculations is obtained with our model with a fixed energy dissipated per contact $\Delta E=56 \mathrm{eV}$. We should stress that this agreement does not specifically support the simple linear force model. Any arbitrary $F_{\text {hys }}$, acting whenever the tip-sample distances reaches a minimum, would produce similar results: For high free amplitudes ( $A_{0}>40 \mathrm{~nm}$ for this experiment), as the tip approaches the sample the system enters abruptly into the repulsive regime $\left(\mathrm{RR}, \varphi<90^{\circ}\right)$. Whenever the system is in the RR the dissipation is given by $P_{\text {dis }}=\Delta E v_{0}$ as expected. Starting from lower $A_{0}$ the system remains in the attractive regime $\left(\mathrm{AR}, \varphi>90^{\circ}\right)$ while the sample approaches the tip. For these $A_{0}$ the dissipated power shows a maximum which increases for increasing $A_{0}$. Similar qualitative phenomena have been predicted for capillary interactions [8] and are 
a direct consequence of a beating phenomenon [15] (see the inset in Fig. 2): In the attractive regime, after the contact process, the cantilever, which has lost energy, will not reach the same amplitude as before the contact, and the tip may not hit the sample surface during the next swings. The power dissipation is then lower than expected.

5 Conclusions We have presented both experimental and theoretical results showing that power dissipation as measured in AM-AFM is not always proportional to the energy dissipated in the tip-sample interaction process. We have shown that adhesive hysteresis modifies the dynamics of the cantilever leading to different dynamic-dissipation curves in the repulsive and attractive regimes.

Acknowledgements This work has been supported by the Spanish M.E.C. (Projects CTQ2005-07993-C0202/BQU, NAN2004-09125-C07-02 and FIS2006-11170C02-02) and by the EU IP "Molecular Imaging" (LSHG-CT-2003-503259). M.K., M.F. and M.L. acknowledge financial support from the MEC.

\section{References}

[1] Fundamentals of Friction: Macroscopic and Microscopic Processes, Edited by I.L. Singer and H.M. Pollock, NATO ASI Series E: Applied Sciences, Vol. 220 (Kluwer, Dordrecht, 1992).

[2] J.P. Cleveland, B. Anczykowski, A.E. Schmid, and V.B. Elings, Appl. Phys. Lett. 72, 2613 (1998).

[3] J. Tamayo and R. García, Appl. Phys. Lett. 73, 2926 (1998).

[4] P.D. Ashby and C.M. Lieber, J. Am. Chem. Soc. 127, 6814 (2005).

[5] R. Garcia, R. Magerle and R. Pérez, Nature Materials 6, 405 (2007).

[6] P. Gleyzes, P. K. Kuo, and A. C. Boccara, Appl. Phys. Lett. 58, 2989 (1991).

[7] B. Anczykowski, D. Krüger, and H. Fuchs, Phys. Rev. B 53, 15485 (1996).

[8] E. Sahagún, P. García-Mochales, G.M. Sacha, and J.J. Sáenz, Phys. Rev. Lett. 98, 176106 (2007).

[9] J.E. Sader, J.W.M. Chon, and P. Mulvaney, Rev. Sci. Instrum. 70, 3967 (1999).

[10] B.V. Derjaguin, V.M. Muller, Y.P. Toporov, J. Colloid Interface Sci. 53,314 (1975).

[11] R. García and A. San Paulo, Phys. Rev. B 60, 4961 (1999).

[12] Y.L. Chen, C.A. Helm and J.N. Israelachvili, J. Phys. Chem. 95, 10736 (1991).

[13] U. Landman, W.D. Luedtke, N.A. Burnham and R.J. Colton, Science 248, 454 (1990).

[14] U. Dürig, New J. Phys. 2, 5 (2000); C. Bernard, S. Marsaudon, R. Boisgard and J.-P. Aimé, Nanotechnology 19, 035709 (2008). 